\title{
The Equations of Motion for Thermally Driven, Buoyant Flows
}

\author{
Ronald G. Rehm* and Howard R. Baum $†$ \\ National Bureau of Standards, Washington, D. C. 20234 \\ January 17, 1978
}

In this paper a set of approximate equations is derived which is applicable to very nonadiabatic, nondissipative, buoyant flows of a perfect gas. The flows are assumed to be generated by a heat source in which the heat is added slowly. The study is motivated by the occurrence of such flows in fires. There, the time scale associated with the fire growth and resultant fluid motion is usually long compared with the transit time of an acoustic signal (based on the temperature derived from the heat added) across the spatial extent of the fire. The appro' mate equations are characterized by a spatially uniform mean pressure appearing in both the energy equa on and the equation of state with the spatially nonuniform portion of the pressure only appearing in the momentum equation. Therefore, the pressure remains almost constant in space while significant density and temperature variations, such as might occur in a fire, are allowed. The approximate equations are shown to reduce to the Boussinesq equations when the heat addition is mild. These equations are also shown in general to admit internal-wave motions while "filtering out" high-frequency, acoustic waves. In addition, they are shown to be expressible in conservation form, the pressure satisfying an elliptic equation whose homogeneous terms are derivable from the wave equation by letting the sound speed become infinite. An equation for the mean pressure is also obtained. For the special case of a room heated at a uniform rate with a small leak to the outside, an approximate solution for the mean pressure is determined explicitly.

Key words: Buoyant flow; fire research; gravity-driven flows; model equations; nonadiabatic flow.

\section{Introduction}

The motions considered in this paper are those which arise solely due to localized addition of heat to an otherwise quiescent fluid in the presence of gravity. The principal application of interest to the authors is the movement of smoke and hot gases caused by fires. However, the formalism introduced will be appropriate to rederive equations previously used to describe rapid heating by a laser and equations which describe detonations, as well as those appropriate to fire research.

The purpose of this paper is to obtain formally a set of equations of motion which permit description of large temperature and density variations due to volumetric heat addition without requiring a simultaneous description of acoustic oscillations arising because of the elastic properties of the fluid. Such model equations include the important features of buoyant flows without requiring excessive computer time necessary to determine high-frequency sound waves when numerically integrated. In this sense the equations "filter out" the sound waves while describing the lower frequency, organized motions due to buoyant effects such as internal waves.

Such filtering is analogous to that employed previously to eliminate acoustic waves from the equations of motion in studies of atmospheric dynamics [1-4]. However, both the procedure used to implement the filtering and the final equations are quite different. Equations closely related to those derived in the present study have been used in attempts to describe both steady-state natural convection over a hot horizontal surface [5] and transient two-phase flow arising in nuclear reactor research $[6,7]$. In each of these studies, large density variations due to temperature changes are admitted, but compressibility effects are suppressed. In the reactorrelated papers, the liquid-vapor mixture is modeled as a thermally expanding, homogeneous fluid, and the discussions are closely tied to computer code development. In neither case has the effect of a closed environment on the pressure been considered in detail (see sec. 5). To the authors' knowledge, moreover, no systematic derivation of these equations has been presented previously.

This derivation is different than that used when considering most atmospheric studies. Although the atmosphere is indeed thermally driven, the sources of heat are sufficiently diffuse that direct heating is

\footnotetext{
* Institute for Basic Standards.

${ }^{1}$ Figures in brackets indicate literature references at the end of this paper.
} 
normally neglected, its principal effect being to stratify the atmosphere. The induced motions are in fact usually assumed to be nearly adiabatic disturbances to a pre-stratified state of rest (see ref. 1, 8 and 9 for example). This stands in direct contrast to the nearly isobaric processes in any initially (nearly) unstratified environment of interest in fire research.

Another difference is the role played by the Boussinesq equations. Considerable attention has been given in the geophysical and astrophysical literature to the conditions under which these equations may be used [10-12]. By contrast, their utility in fire research seems to the present authors to be rather limited, although many useful studies based on them have been performed. The equations derived below contain all the terms retained in the Boussinesq equations. However, the large variations in density caused by the local heat addition prevent the linearization of density about any nominal, unperturbed value.

The fluid is taken to be an inviscid non-heat conducting perfect gas. The magnitude and the spatial and temporal variation of the heat source are taken as known. These approximations are justified because under conditions characterisitic of even a small room fire, the Grashof numbers (representing the ratio of the inertial to viscous forces for natural convection) are large enough for molecular transport phenomena to be important only in the highly convoluted flame sheets which constitute the region of intense heat addition. The study of the detailed flame structure of real fires is an extraordinarily complicated subject in its own right, and is bypassed here by specifying the heat source. Batchelor [16] gives a brief but relevant discussion of the applicability of the inviscid equations in the context of atmospheric motions. It should be noted that such simplifications do not preclude a description of turbulence; but no turbulence model is explicitly included in this study. Simple models of smoke and hot gas transport which neglect molecular transport phenomena have been reasonably successful in predicting global properties of flow fields [17]. The present work is intended as a first step towards more detailed studies along these lines.

In the next section the model equations are derived using a dimensionless formulation based upon the characteristics of the (volumetric) heat source. In section 3 some of the properties of this system of equations are examined. Particularly, it is shown that internal waves will be described while acoustic waves are not permitted by this approximate set of equations. Also, the equations are rewritten in conservation form, a form often preferred for numerical computations, and the mixed hyperbolic, elliptic nature of the equations is noted. In the fourth section a discussion is given to show how the Boussinesq equations are obtained as a special case of these equations. In the final section, an equation for the mean pressure is obtained. This equation is novel and is a direct result of the filtering analysis performed in section 2. An approximate solution is obtained for the special case of an enclosure heated at a uniform rate with a small leak to the outside.

\section{Formulation}

The equations of motion for a perfect gas in the presence of an energy source of strength $Q$ may be written in the form:

$$
\begin{gathered}
\frac{\partial \rho}{\partial t}+\frac{\partial}{\partial x_{i}}\left(\rho u_{i}\right)=0 \\
\rho\left(\frac{\partial u_{i}}{\partial t}+u_{k} \frac{\partial u_{i}}{\partial x_{k}}\right)+\frac{\partial p}{\partial x_{i}}-\rho g n_{i}=0 \\
\rho C_{p}\left(\frac{\partial T}{\partial t}+u_{k} \frac{\partial T}{\partial x_{k}}\right)-\left(\frac{\partial p}{\partial t}+u_{k} \frac{\partial p}{\partial x_{k}}\right)=Q \\
p=\rho \tilde{R} T
\end{gathered}
$$

Here $\rho$ is the density, $p$ the pressure, $T$ the temperature, and $u_{i}$ the velocity at any position $x_{i}$ and time $t$. Also, $\tilde{R}$ is the gas constant, $C_{p}$ the constant-pressure specific heat, and $n_{i} g$ is the gravitational acceleration.

The fundamental assumption on which this paper is based is that the source strength may be usefully characterized in the form: 


$$
Q=\frac{E_{o}}{t_{o}} \tilde{Q}\left(\frac{x_{i}}{l}, \frac{t}{t_{o}}\right)
$$

Thus $E_{o}$ is a dimensional constant which determines the strength of the source. The quantity $\dot{Q}$ is a function of order unity which varies smoothly with respect to its arguments. The independent variables $x_{i}$ and $t$ are now made dimensionless with respect to $l$ and $t_{o}$ respectively, where $l$ and $t_{o}$ are the length and time scales describing the spatial extent and the temporal variation of the heat source. The dependent variables are scaled as follows:

$$
\begin{aligned}
\rho & =\rho_{o} R\left(y_{i}, \tau\right) \\
T & =\frac{E_{o}}{\rho_{o} C_{p}} \theta\left(y_{i}, \tau\right) \\
u_{i} & =\left(\frac{\gamma-1}{\gamma} \frac{E_{o}}{\rho_{o}}\right)^{1 / 2} V_{i}\left(y_{i}, \tau\right) \\
p & =\frac{\gamma-1}{\gamma} E_{o} P\left(y_{i}, \tau\right) \\
x_{i} & =l y_{i}, t=t_{o} \tau .
\end{aligned}
$$

The temperature scaling used in eq (3) is chosen so that the time derivatives in the energy equation always are the same magnitude as the source term. The density is normalized with respect to an ambient level $\rho_{o}$ which occurs in the absence of any heat addition. The pressure scale then follows from the equation of state. The velocity is non-dimensionalized with respect to a thermal (sound) speed based on the temperature scale. The specific heat ratio is denoted by $\gamma$.

Substituting eqs (2) and (3) into eq (1); the dimensionless equations of motion are:

$$
\begin{gathered}
\frac{\partial R}{\partial \tau}+\delta \frac{\partial}{\partial y_{i}}\left(R V_{i}\right)=0 \\
R\left\{\frac{\partial V_{i}}{\partial \tau}+\delta\left[V_{j} \frac{\partial V_{i}}{\partial y_{j}}\right]\right\}+\delta \frac{\partial P}{\partial y_{i}}=\frac{g t_{o}^{2}}{l} \frac{1}{\delta} n_{i} R \\
R\left\{\frac{\partial \theta}{\partial \tau}+\delta V_{j} \frac{\partial \theta}{\partial y_{j}}\right\}-\frac{\gamma-1}{\gamma}\left\{\frac{\partial P}{\partial \tau}+\delta V_{j} \frac{\partial P}{\partial y_{j}}\right\}=\hat{Q}\left(y_{i}, \tau\right) \\
p=R \theta
\end{gathered}
$$

The parameter $\delta$ is defined by

$$
\delta=\frac{t_{o}}{l}\left(\frac{\gamma-1}{\gamma} \frac{E_{o}}{\rho_{o}}\right)^{\frac{1}{2}} .
$$

This parameter plays a fundamental role in what follows. Physically, it represents the distance, based on the characteristic velocity $\left(\left(\frac{\gamma-1}{\gamma}\right) E_{o} / \rho_{o}\right)^{\frac{1}{2}}$, a disturbance can travel during the time the source changes appreciably divided by the spatial extent of the source. The magnitude of $\delta$ is directly related to the heating rate. The case $\delta \ll 1$ corresponds to rapid heat addition, realized in laser heating devices. The case $\delta \sim 0$ (1), an intermediate case, describes detonation phenomena. Finally, the case $\delta \gg 1$, corresponding to slow heat addition, occurs in both controlled combustion and in fires.

The mathematical task now reduces to displaying the dependence of the reduced variables defined in eq (3) upon $\delta$ in the three cases described above. The dominant terms in the expansions of these variables as functions of $\delta$ then satisfy equations which are subsets of eq (4). The expansions, and thus the resulting equations, are different for each case. They will now be treated in order. 
(i) Fast heat addition $(\delta \ll 1)$.

The dependent variables may be expanded in the form:

$$
\begin{gathered}
P=P^{(o)}\left(y_{i}, \tau\right)+0(\delta) \\
R=R^{(o)}\left(y_{i}, \tau\right)+0(\delta) \\
\theta=\theta^{(o)}\left(y_{i}, \tau\right)+0(\delta) \\
V_{i}=\delta\left\{V_{i}^{(o)}\left(y_{j}, \tau\right)+0(\delta)\right\} .
\end{gathered}
$$

Substituting the expansion (5) into eqs (4), the equations of motion reduce to:

$$
\begin{gathered}
\frac{\partial R^{(o)}}{\partial \tau}=0 \\
R^{(o)} \frac{\partial V_{i}^{(o)}}{\partial \tau}+\frac{\partial P^{(o)}}{\partial y_{i}}=\frac{\rho_{o} g l}{E_{o}}\left(\frac{\gamma}{\gamma-1}\right) n_{i} R^{(o)} \\
R^{(o)}\left[\frac{\partial \theta^{(o)}}{\partial \tau}-\frac{\gamma-1}{\gamma}-\frac{\partial P^{(o)}}{\partial \tau}\right]=\tilde{Q}\left(y_{i}, \tau\right) \\
P^{(o)}=R^{(o)} \theta^{(o)} .
\end{gathered}
$$

Equations (6) are a slight generalization of those used by Rehm [11] to study the laser bleaching wave. Note that once the heat addition model for $\tilde{Q}$ is specified in terms of the thermodynamic variables and their gradients, the momentum equations are decoupled from the remaining three of eqs (6). Moreover, the density remains constant. This situation arises because the gas has not moved appreciably during the short interval in which the heat is added. The quantity $\rho_{\mathrm{o}} g l / E_{o}$ is usually quite small in laser applications.

(ii) Intermediate heat addition $(\delta \sim 0(1))$.

When $\delta$ is $0(1)$, no terms of eq (4) (except possibly those due to gravitation) can be ignored. In this case, it is easier to put $\delta=1$. This is equivalent to replacing the length scale $l$ by $t_{o}\left(\frac{\gamma-1}{\gamma} \frac{E_{o}}{\rho_{o}}\right)$. This is a mathematical statement of the fact that the ratio of the length scale to the time scale is determined by the wave speed in a detonation [14]. The speed of such a front is of the same order as the thermal speed $\left(\frac{\gamma-1}{\gamma} \frac{E_{o}}{\rho_{0}}\right)^{\frac{1}{2}}$.

(iii) Slow heat addition $(\delta \gg 1)$.

Since $\delta$ is large, an expansion in negative powers of $\delta$ is anticipated. Inspection of the energy equation shows that the spatial convection terms are too large by a factor $\delta$ to be driven by the source term. If the velocity is reduced by a factor $\delta^{-1}$, the momentum balance requires the pressure gradients be reduced by a factor $\delta^{-2}$. The expansion then becomes:

$$
\begin{aligned}
V_{i} & =\delta^{-1}\left\{V_{i}^{(o)}+0\left(\delta^{-2}\right)\right\} \\
P & =P^{(o)}(\tau)+\delta^{-2} P^{(1)} \\
\theta & =\theta^{(o)}+0\left(\delta^{-2}\right) \\
R & =R^{(o)}+0\left(\delta^{-2}\right) .
\end{aligned}
$$

The suppressed arguments of all dependent variables except $P^{(o)}(\tau)$ are both $y_{i}$ and $\tau$. Substitution of the forms (7) into eqs (4) yields: 


$$
\begin{gathered}
\frac{\partial R^{(o)}}{\partial \tau}+\frac{\partial}{\partial y_{i}}\left(R^{(o)} V_{i}^{(o)}\right)=0 \\
R^{(o)}\left\{\frac{\partial V_{i}^{(o)}}{\partial \tau}+V_{j}^{(o)} \frac{\partial V_{i}^{(o)}}{\partial y_{j}}\right\}+\frac{\partial P^{(1)}}{\partial y_{i}}-n_{i} \frac{g t_{o}^{2}}{l} R^{(o)}=0 \\
R^{(o)}\left\{\frac{\partial \theta^{(o)}}{\partial \tau}+V_{j}^{(o)} \frac{\partial \theta^{(o)}}{\partial y_{j}}\right\}-\frac{\gamma-1}{\gamma} \frac{d P^{(o)}}{d \tau}=\tilde{Q} \\
R^{(o)} \theta^{(o)}=P^{(o)}(\tau) .
\end{gathered}
$$

Note that the expansion (7) implies that the spatial variations in pressure are extremely small. The reference pressure level $P^{(o)}(\tau)$ can be determined from the boundary conditions. For example, the pressure variation in an enclosed volume $W$ subject to heating is given by (see sect. 5 for a more detailed derivation):

$$
\frac{1}{\gamma} W \frac{d P^{(o)}}{d \tau}=\int_{W} \tilde{Q} d^{3} y
$$

If the domain is open to the atmosphere, a point can be found at which the pressure is specified as $P_{\infty}$, say. Then:

$$
P^{(o)}=\frac{\gamma}{\gamma-1} \frac{P_{\infty}}{E_{o}}
$$

This reference pressure point can be taken as the origin of the vertical coordinate $y_{i} n_{i}$ without loss of generality.

If the quantity $g t_{o}^{2} / l \ll 1$, then the gravitational force may be neglected. This situation arises frequently in studies of nearly isobaric combustion processes [13]. However, in fire research there are many problems for which $\alpha \equiv g t_{o}^{2} / l$ is $O(1)$. It is then convenient to redefine the time scale $t_{o}$ such that $\alpha=1$. The velocities are then $O\left((g l)^{\frac{1}{2}}\right)$ in magnitude, while the spatial pressure perturbation is $O(\rho g l)$ and the temperature is $0\left\{\frac{E_{o}}{\rho_{o} C_{p}}\right\}$

In dimensional form, the approximate equations of motion (eqs (8)) are:

$$
\begin{gathered}
\frac{\partial \rho}{\partial t}+\frac{\partial}{\partial x_{j}}\left(\rho u_{j}\right)=0 \\
\rho\left(\frac{\partial u_{i}}{\partial t}+u_{j} \frac{\partial u_{i}}{\partial x_{j}}\right)+\frac{\partial\left(p-p_{o}\right)}{\partial x_{i}}-\rho n_{i} g=0 \\
\rho C_{P}\left(\frac{\partial T}{\partial t}+u_{j} \frac{\partial T}{\partial x_{j}}\right)-\frac{d p_{o}}{d t}=Q \\
p_{o}(t)=\rho R T .
\end{gathered}
$$

Since $p_{o}$ is necessarily independent of position, it follows from the equation of state that the ambient atmosphere in the absence of heating must be taken as having a constant density. This limitation is ultimately traceable to the assumption stated earlier that the vertical extent of the source is much less than the scale height of the atmosphere. It is often convenient to replace the variable $p-p_{o}$ by a reduced pressure $p^{*}$, defined by:

$$
p^{*}=p-p_{o}-\rho_{0} g x_{i} n_{i}
$$


Equations (11) are then altered in that $\rho$ must be replaced by $\rho-\rho_{0}$ in the gravitational force term and $p-p_{o}$ replaced by $p^{*}$ in the pressure gradient term. With this transformation, eqs (11) appear more similar to the Boussinesq equations.

\section{Properties of the Derived Equations}

In this section two properties of eqs (11) will be examined. First, it will be demonstrated that these equations contain buoyant effects without including the elastic effects of the medium. This is demonstrated by examining small-amplitude waves and showing that internal waves (buoyant effects) are allowed in this description while acoustic waves (elastic effects) are excluded. Second, the conservation form of eqs (11) are examined and the mixed hyperbolic and elliptic nature of these equations is noted explicitly.

To demonstrate the first property, we assume that the flow has evolved to point where, in eqs (11), the mean thermodynamic variables are stratified so that, $\rho=\rho_{o o}(z)$ and $T=T_{o o}(z)$ while $p=p_{o}=$ constant. (For convenience, the spatial coordinates will be denoted by $x, y$ and $z$.) All the flow variables are perturbed about these quantities. For simplicity, the heat source is taken to be zero. Also, we will take the buoyancy frequency $N^{2} \equiv-g \frac{d \rho_{o o}}{d z} / \rho_{o o}$ to be constant (so that the density is stratified exponentially). The type of waves described by eqs (11) is not changed by these simplifications. The equations (with variable $N^{2}(z)$ ) would describe the fluctuations produced in a closed room following a fire which had stably stratified the environment outside the buoyant plume and then gone out after consuming the available oxygen supply.

The perturbed dependent variables are substituted into eqs (11), and the equations are linearized in the perturbation quantities. The temperature perturbation can be eliminated from the resulting equations.

From the momentum equations and the continuity equation, the velocities can then be eliminated to give an equation coupling the density and pressure perturbations.

From the energy equation and $z$-component of the momentum equation, the vertical velocity can be eliminated to give a second equation coupling the density and pressure perturbations. These two coupled equations can then be used to obtain a single equation for the perturbation pressure $\tilde{p}$ :

$$
\left[\left(\frac{\partial^{2}}{\partial t^{2}}+N^{2}\right)\left(\frac{\partial^{2}}{\partial x^{2}}+\frac{\partial^{2}}{\partial y^{2}}\right)+\frac{\partial^{2}}{\partial t^{2}} \frac{\partial^{2}}{\partial z^{2}}+\frac{N^{2}}{g} \frac{\partial^{2}}{\partial t^{2}} \frac{\partial}{\partial z}\right] \tilde{p}=0 .
$$

The dispersion relation is obtained by assuming waves of the form

$$
\tilde{p}=A \exp \left[-\frac{N^{2}}{2 g} z+i\left(x k_{x}+y k_{y}+z k_{z}-\omega t\right)\right] .
$$

Then

$$
-\left[k_{z}^{2}+\left(\frac{N^{2}}{2 g}\right)^{2}\right]+\frac{N^{2}-\omega^{2}}{\omega^{2}}\left(k_{x}^{2}+k_{y}^{2}\right)=0 .
$$

This dispersion relation is the one which describes internal gravity waves. For the two-dimensional case (i.e. when there is no dependence of the motion in the $y$-direction), the equation was derived and the waves discussed, for example, by Lamb [18], by Mowbray and Rarity [19] and by Whitham [21]. The equations do not permit acoustic waves, as claimed earlier. In the derivations referenced above, the starting equations are taken to be those appropriate for an incompressible fluid. The dispersion relations and properties of more general acoustic-gravity waves in a compressible, stratified medium are discussed in books by Eckart [8], and by Yih [20] and by Dutton [9] for example.

It is important to note that the model equations contain only internal waves and not acoustic waves. Apparently some confusion has existed in the past concerning what assumptions are necessary to assure this filtering. For example, in the paper by Gough [4], where the "anelastic approximation" is derived and discussed, the continuity equation is replaced by $\boldsymbol{\nabla} \cdot(\rho \mathbf{u})=0$, and it is stated that this approximation is necessary in order to remove the acoustic modes of oscillation. As seen above, the time derivative of the 
density in the continuity equation need not be eliminated, however. Rather, acoustic modes are removed when the pressure in the momentum equation is decoupled from density and temperature fluctuations arising through the equation of state.

Many numerical methods for solving fluid mechanics problems are based on finite difference schemes obtained from conservation forms of the equations of motion. The appropriate forms of the continuity and momentum equations are given by:

$$
\begin{gathered}
\frac{\partial \rho}{\partial t}+\frac{\partial}{\partial x_{i}}\left(\rho u_{i}\right)=0 \\
\frac{\partial}{\partial t}\left(\rho u_{i}\right)+\frac{\partial}{\partial x_{j}}\left(\rho u_{i} u_{j}\right)+\frac{\partial p}{\partial x_{i}}-\rho g_{i}=0 .
\end{gathered}
$$

Equation (16) may be thought of as allowing the computation of $\rho$ and $u_{i}$ at a time $t+\delta t$ given information about all quantities at $t$. In order to complete the hypothetical computation of the flow variables at $t+\delta t$, equations for the pressure must be determined. This determination consists of two parts; an equation for the reference level $p_{o}(t)$, and an equation for the spatial distribution of the overpressure $p\left(x_{i}, t\right)$. The equation for $p_{0}(t)$ is derived and discussed in section 5. A solution for this reference level pressure is also presented.

The equation for the overpressure $p$ is obtained by dividing the momentum eq (11) by $\rho$ and taking the divergence of the resulting expression. Then, using the continuity equation:

$$
\begin{gathered}
\frac{\partial}{\partial x_{i}}\left(\frac{1}{\rho} \frac{\partial p}{\partial x_{i}}\right)=-\left\{\frac{\partial}{\partial x_{i}}\left(u_{j} \cdot \frac{\partial u_{i}}{\partial x_{j}}\right)+\frac{\partial \Delta}{\partial t}\right\} \\
\frac{\partial \Delta}{\partial t}=-\frac{1}{\gamma} \frac{d}{d t}\left(\frac{1}{p_{o}} \frac{d p_{o}}{d t}\right)+\frac{\partial}{\partial t}\left(\frac{\gamma-1}{\gamma} \frac{Q}{p_{0}}\right) \\
\Delta=\frac{\partial u_{i}}{\partial x_{i}} .
\end{gathered}
$$

Note that the terms not involving $p$ in eq (17) are all known at time $t+\delta t$. Since the density $\rho$ is also known at $t+\delta t, p$ satisfies a linear self adjoint elliptic equation at each instant of time. The appropriate boundary conditions are a specification of the normal derivative of $p$ obtained from evaluation of the momentum equation at the boundary. Such a specification ensures that the consistency condition for the existence of a solution to eq (17) is satisfied automatically. This equation is the generalization of Poisson's equation appropriate to an inhomogeneous fluid. It remains an elliptic equation, despite the density variations, because the sound waves which would lead to a finite propagation speed (and hence to a hyperbolic system of equations) have been eliminated.

This can be seen quite readily if the steps leading to eq (17) are performed on the full equations (eqs (1)) rather than the reduced set (eqs (11)). The result is:

$$
\begin{aligned}
\frac{\partial}{\partial x_{i}}\left(\frac{1}{\rho} \frac{\partial p}{\partial x_{i}}\right)-\frac{1}{\rho C^{2}} \frac{\partial^{2} p}{\partial t^{2}}=\frac{1}{\gamma p}\left[\frac{\partial}{\partial t}\left(u_{i} \frac{\partial p}{\partial x_{i}}\right)-\frac{1}{p} \frac{\partial p}{\partial t}\left(u_{i} \frac{\partial p}{\partial x_{i}}\right)\right]- & \\
& {\left[\frac{1}{\gamma \mathrm{p}^{2}}\left(\frac{\partial \mathrm{p}}{\partial \mathrm{t}}\right)^{2}+\frac{\gamma-1}{\gamma} \frac{\partial}{\partial t}\left(\frac{Q}{p}\right)+\frac{\partial}{\partial x_{i}}\left(u_{j} \frac{\partial u_{i}}{\partial x_{j}}\right)\right] . }
\end{aligned}
$$

The quantity $C^{2}$ in eq (18) is the square of the local sound speed. Equation (18) is the wave equation for the pressure, a hyperbolic equation generalizing eq (17). The terms in the second brackets on the right hand side above, together with the term $\frac{1}{\rho C^{2}} \frac{\partial^{2} p}{\partial t^{2}}$, also appear on the right hand side of eq (17).

Equation (18) can be reduced formally to eq (17) by nondimensionalization and expansion of the dependent variables (retaining only terms $0(1)$ and neglecting $0\left(\delta^{-1}\right)$ ) as in section 2 . When this is done, terms linear in 
pressure gradients and quadratic in velocities must be retained. Products of pressure gradients and velocities are of higher order and may be ignored. Then eq (18) reduces to eq (17).

The overall system of equations is neither elliptic nor hyperbolic. The mixed character of the equations may be seen by noting that the energy equation (eq (11)) is a hyperbolic equation for $T$. Indeed, the overall system may be either elliptic or hyperbolic (or neither) depending on the problem under consideration.

The variable density coefficient in the pressure equation complicates the task of numerical solution. However, relatively efficient schemes for handling equations of this type have been developed [22]. Thus, practical finite difference computational schemes based on eqs (16), (17) and an equation for the mean pressure (discussed in sect. 5) are feasible.

\section{The Boussinesq Equations}

The derivation presented in section 2 is based on two assumptions. First, the length scale, time scale and the temperature scale associated with the volumetric heat source are such that the heat addition is slow. This assumption implies that the pressure over a large region surrounding the source is almost uniform in space (while it may vary with time) during heating. However, it does not imply any restriction upon the magnitude of the density (or temperature) variation during heating. The second assumption is that the flow velocities are induced by buoyant effects. This assumption relates the magnitude of the temperature variation, the density variation and the flow velocities induced by the heat source.

Batchelor, in obtaining conditions for which atmospheric motions are determined only by an overall Richardson number (the ratio of buoyancy to inertia forces), has discussed the possibility of atmospheric motions where the pressure varies only slightly from hydrostatic, while the density and temperature deviate significantly from hydrostatic values [16]. Batchelor was concerned explicitly with adiabatic flows. However, for nonadiabatic motions the same possibility exists when heat is released slowly, as shown by the derivation in section 2.

The Boussinesq equations arise when two additional assumptions are made. The first assumption is that the scale height associated with the static density variation (the density variation in the absence of motions) is much larger than the vertical length scales of interest. Then the static density variation from its mean value is small. The second assumption is that the density variations produced by the heat source are small. These are the assumptions made by Speigel and Veronis [10] in their derivation of the Boussinesq equations.

The height scale associated with hydrostatic variations in the thermodynamic variables was already assumed to be much larger than the vertical length of interest in section 2 . Therefore, the spatial variations in temperature and density (as well as in pressure) in the absence of motion were taken as small, satisfying the first assumption stated by Speigel and Veronis. (In cases of practical interest in fire research, the hydrostatic variations of these quantities are negligible compared with variations induced by the heat source.) The second assumption, that motion-related temperature and density variations are small, implies that the temperature-

variation scale $\frac{E_{o}}{\rho_{o} C_{p}}$ is small compared with the nominal ambient temperature $T_{o}$ : hence we assume $\frac{E_{o}}{\rho_{o} C_{p} T_{o}} \equiv$ $\epsilon \ll 1$.

With these additional assumptions (and a change in the time scale discussed below), the Boussinesq equations can be derived formally from eqs (8) by expanding the dimensionless dependent variables in a series of powers of $\epsilon$. (This second expansion implies that the Boussinesq equations are obtained by a twoparameter expansion of dependent variables in terms of parameters $1 / \delta$ and $\epsilon$.) However, rather than performing this second expansion, which is very formal and notationally cumbersome, we demonstrate how the Boussinesq equations are obtained from the dimensional equations, (11), in a more physically meaningful way.

As suggested in section 2, we introduce the reduced pressure $p^{*}$ defined in eq (12). We also define a reduced density $\rho^{*}$ and a reduced temperature $T^{*}$ as follows:

$$
\begin{aligned}
& \rho^{*}=\rho-\rho_{o} \\
& T^{*}=T-T_{o} .
\end{aligned}
$$

For simplicity we assume that $p_{o}$ is constant. Introduction of definitions (12) and (19) into Eqs (11) yields 


$$
\begin{array}{r}
\frac{\partial \rho^{*}}{\partial t}+\frac{\partial \rho^{*} u_{j}}{\partial x_{j}}+\rho_{o} \frac{\partial u_{j}}{\partial x_{j}}=0 \\
\left(\rho^{*}+\rho_{o}\right)\left(\frac{\partial u_{i}}{\partial t}+u_{j} \frac{\partial u_{i}}{\partial x_{j}}\right)+\frac{\partial p^{*}}{\partial x_{i}}-\rho^{*} n_{i} g=0 \\
\left(\rho^{*}+\rho_{o}\right)\left(\frac{\partial T^{*}}{\partial t}+u_{j} \frac{\partial T^{*}}{\partial x_{j}}\right)=Q \\
p_{o}=\left(\rho_{o}+\rho^{*}\right)\left(T_{o}+T^{*}\right) \tilde{R} .
\end{array}
$$

The assumption that motion-related temperature and density variations are small implies that the reduced density, temperature and pressure must be proportional to $\epsilon$. Since the buoyant effects drive the flow, the acceleration term in the second of eqs (20) must also be proportional to $\epsilon$. This implies that the time scale for motion is $t_{0}^{\prime}=t_{o} / \epsilon^{\frac{1}{2}}=\left(\frac{l}{\epsilon g}\right)^{\frac{1}{2}}$, and that the velocity scale is proportional to $\epsilon^{\frac{1}{2}}$. Finally, since $Q \propto E_{o} / t_{o}$, the heat source is proportional to $\epsilon^{\frac{3}{2}}$, Therefore, with the formal substitutions

$$
\begin{aligned}
p^{*} & =\epsilon \hat{p} \\
\rho^{*} & =\epsilon \hat{\rho} \\
T^{*} & =\epsilon \hat{T} \\
u & =\epsilon^{\frac{1}{2}} \hat{u} \\
t & =t^{\prime} / \epsilon^{\frac{1}{2}} \\
Q & =\epsilon Q .
\end{aligned}
$$

Equations (20) become, to lowest order in $\epsilon$,

$$
\begin{gathered}
\frac{\partial \hat{u}_{j}}{\partial x_{j}}=0 \\
\rho_{o}\left(\frac{\partial \hat{u}_{i}}{\partial t^{\prime}}+\hat{u}_{j} \frac{\partial \hat{u}_{i}}{\partial x_{j}}\right)+\frac{\partial \hat{p}}{\partial x_{i}}-\hat{\rho} n_{i} g=0 \\
\rho_{o}\left(\frac{\partial \hat{T}}{\partial t^{\prime}}+\hat{u}_{j} \frac{\partial \hat{T}}{\partial x_{j}}\right)=\hat{Q} \\
\frac{\hat{\rho}}{\rho_{o}}+\frac{\hat{T}}{T_{o}}=0
\end{gathered}
$$

where $p_{o}=\tilde{R} \rho_{o} T_{o}$.

These are the equations, in the absence of dissipative effects, usually described as the Boussinesq equations: the density is considered to be constant except where the density difference produces a buoyant force [10].

\section{The Mean Pressure}

The variakle mean pressure level $p_{0}(t)$ is determined from the approximate system of eqs (11) by a consistency argument. The continuity equation (the first of eqs (11)) may be multiplied by $C_{P} T$ and added to the energy equation (the third of eqs (11)) to obtain:

$$
\frac{\gamma}{\gamma-1} p_{o}(t) \frac{\partial u_{k}}{\partial x_{k}}+\frac{1}{\gamma-1} \frac{d p_{o}}{d t}=Q .
$$


As mentioned earlier if the domain under consideration is open to the ambient atmosphere, then $p_{o}$ is constant and need not be considered further. Now let the domain be a "closed" volume $V$ (the meaning of "closed" will be made clear) bounded by a surface A. Integration of eq (23) over $V$ yields:

$$
\left\{\frac{\gamma}{\gamma-1} \int u_{i} n_{i} d A\right\} p_{o}(t)+\frac{V}{\gamma-1} \frac{d p_{o}}{d t}=\int_{V} Q d V .
$$

Equation (24) is a differential equation for $p_{0}(t)$ whose coefficients are determined by the geometry of the enclosure and boundary conditions specifying the normal component of the velocity on the boundary. Thus, $p_{o}(t)$ is determined by the requirement that the net efflux of fluid from the enclosure be consistent with the net rate of heat addition. Note that if the room were truly closed, in the sense that $u_{i} n_{i}=0$ at each point on the boundary, the mean pressure would rise linearly for a constant heating rate. This would very quickly lead to pressures sufficiently high to cause structural collapse. Most rooms in buildings are not closed in this sense, even with doors and windows shut. A more realistic geometry will be discussed below.

Consider a room of volume $V$ whose general shape is like that illustrated in the insert in figure 1 . The room is open to the ambient atmosphere at pressure $\mathrm{p}_{\infty}$ only through a crack of length $l$ and height $d$. The aspect ratio $l / d$ and the Reynolds number of the crack are assumed to be sufficiently large for the flow in the crack to be effectively two dimensional and inviscid. The crack is taken to be near the floor but away from the fire. The density of the gas exiting through the opening will then be the ambient value $\rho_{\infty}$ until the hot ceiling layer formed by the fire plume descends to the floor. Under these circumstances, the efflux is given by the usual orifice formula [23]

$$
\int u_{i} n_{i} d A=\frac{\pi}{\pi+2} l d\left\{\frac{2}{\rho_{\infty}}\left(p_{o}(t)-p_{\infty}\right)\right\}^{\frac{1}{2}} .
$$

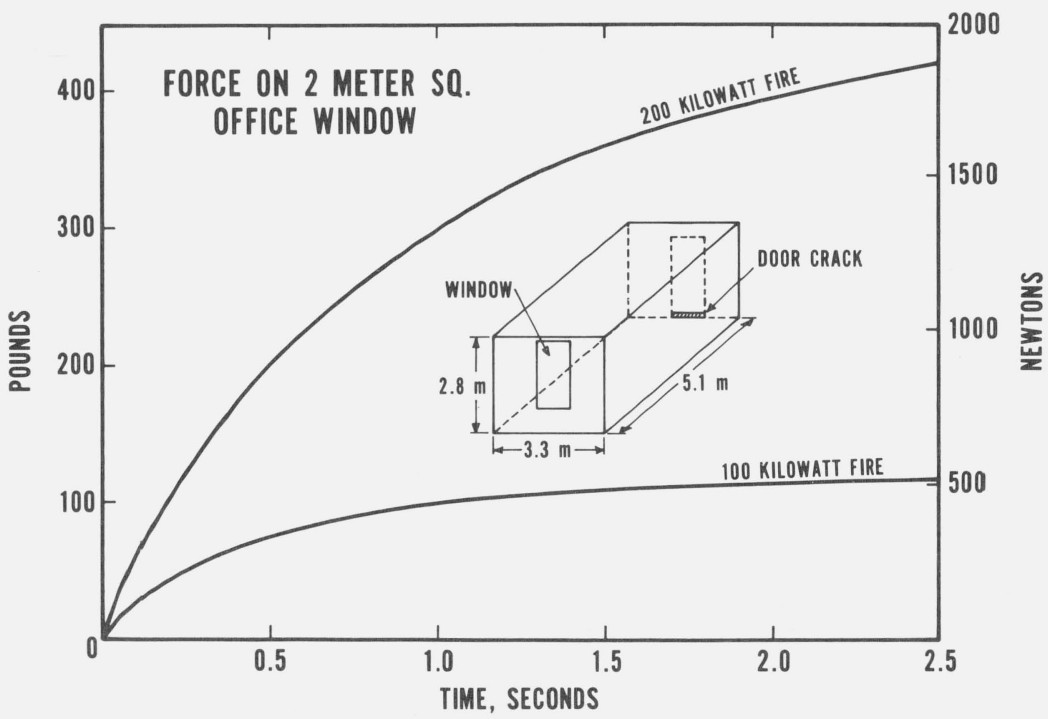

FIGURE 1. The force on a two square meter window caused by the mean pressure rise in a room during a fire. The fire sizes correspond to a small and a large waste-container fire while the room dimensions are those of a typical N.B.S. office. The room is assumed to be closed except for a crack of dimensions $75 \mathrm{~cm}$ by $2.5 \mathrm{~cm}$.

The total rate of heat release for various burning materials is a frequently measured parameter in fire research. A useful compilation is given in ref. 17 and the references therein. For the present calculation this quantity will be characterized as follows:

$$
\int Q d V=\dot{Q}
$$


The quantity $\dot{Q}$ will be taken as the total rate of heat release due to the fire. The losses due to heat transfer to the boundaries are unimportant on the time scale associated with the pressure buildup, although ultimately they are a substantial fraction of $\dot{Q}$.

It is convenient to introduce non dimensional variables for $p_{o}$ and $t$.

$$
\begin{aligned}
p_{o}(t) & =p_{\infty}\left\{1+\hat{\boldsymbol{\epsilon}}^{2} P(\tau)\right\} \\
\hat{\boldsymbol{\epsilon}} & =\frac{\gamma-1}{\sqrt{2 \gamma}}\left(\frac{\pi+2}{\pi}\right) \frac{\dot{Q}}{l d C_{\infty} p_{\infty}} \\
t & =\left(\frac{\pi+2}{\pi l d C_{\infty}}\right)^{2} \frac{(\gamma-1) \dot{Q} V}{\gamma p_{\infty}} \tau .
\end{aligned}
$$

The quantity $C_{\infty}$ in eq (27) is the sound speed based on the ambient pressure and density. The parameter $\hat{\boldsymbol{\epsilon}}$ determines the maximum steady state pressure rise attainable for a given fire size and enclosure geometry. For most realistic scenarios, $\hat{\epsilon} \ll 1$. Substitution of eqs (25)-(27) into eq (24) and ignoring terms $0\left(\hat{\epsilon}^{2}\right)$ leads to the evolution equation for $\mathrm{p}(\tau)$

$$
\frac{d P}{\mathrm{~d} \tau}=1-\sqrt{ } P .
$$

The solution is readily obtained in the form

$$
\tau=\log (1-\sqrt{ } P)^{-1}-\sqrt{ } P .
$$

Note that $P=1$ corresponds to the steady state, so that the final fractional pressure rise measured in atmospheres is equal to $\hat{\boldsymbol{\epsilon}}^{2}$. Thus, the pressure rise is directly proportional to $\dot{Q}^{2}$ and inversely proportional to the square of the opening area. Although eq (29) can be plotted as a single curve, it is more instructive to pick a specific geometry and display the results in physical units as in figure 1 . The fire sizes chosen correspond to a small and a large waste container fire [17], while the room dimensions are those of a typical NBS office. The crack dimensions are taken to be $75 \mathrm{~cm}$ by $2.5 \mathrm{~cm}$. The quantity $\hat{\epsilon}^{2}$ is approximately 0.01 for the larger fire and the Reynolds number based on crack height is $0\left(10^{4}\right)$ for the smaller fire.

At a time $t=2.5 \mathrm{~s}$ only a few percent of the original room air has left through the crack, so the hot ceiling layer is unlikely to be very deep. Thus, the assumptions underlying the calculation are amply fulfilled. It seems that even a rather unspectacular fire can rapidly generate a considerable unbalanced force on a structure, which can rupture a relatively weak component like a window. This may be at least a partial explanation of the fact, commonly observed, that windows often break in fires.

In summary, the equations derived in section 2 are ones which we feel are important in fire research and may be important in other applications as well. The flows considered are highly nonadiabatic and buoyancy effects are dominant, yet the pressure remains almost uniform spatially as the flow evolves. This implies that the time scale associated with the heat source, which is assumed to be comparable with the time scale for buoyancy effects, is much larger than that time scale required for acoustic signals to equilibrate the pressure over the region of interest. The former time is the one of interest for such flows, and therefore, it is desirable to remove the acoustic waves so that the time step size for numerical integration of the appropriate equations is not limited by the time scale associated with these waves. The equations derived in section 2 accomplish this filtering, as shown in section 3. Furthermore, the equations can be written in conservation form, a form often preferred for numerical computations.

\section{References}

[1] Thompson, P. D., Numerical Weather Analysis and Prediction, Chap. 6 and 11 (The Macmillan Co., New York, N. Y., 1961).

[2] Ogura, Y., and Charney, J. G. A numerical model of thermal convection in the atmosphere, Proc. Internat. Symp. on Numerical Weather Prediction, Tokyo, Japan, Nov. 7-13, 1960, pp. 431-450 (The Meterological Society of Japan, Tokyo, Japan, 1962).

[3] Ogura, Y., and Phillips, N. A., Scale Analysis of Deep and Shallow Convection in the Atmosphere, J. Atmos, Sci. 19, 173-179, (Mar. 1962).

[4] Gough, D. 0., The anelastic approximation for thermal convection, J. Atmos. Sci. 26, pp. 448-456, (May 1969). 
[5] Clarke, J. N., and Riley, N., Natural convection induced in a gas by the presence of a hot porous horizontal surface, Quart. J. Mech. and Appl. Math. 28, Pt. 4, pp. 373-396 (1975).

[6] Porsching, T. A., A finite difference method for thermally expandable fluid transients, Nuclear Sci. and Eng. 64, pp. 177-186, (Sept. 1977).

[7] Stewart, C. W., and George, T. L., A eulerian computation method for fluid flows with large density gradients at all speeds, Nuclear Sci. and Eng. 64, pp. 237-243, (Sept. 1977).

[8] Eckart, C., Hydrodynamics of Oceans and Atmospheres (Pergamon Press, New York, N. Y., 1960).

[9] Dutton, J. A., The Ceaseless Wind, An Introduction to the Theory of Atmospheric Motion (McGraw-Hill Book Co., New York, N. Y., 1976).

[10] Spiegel, E. A., and Veronis, G., On the Boussinesq approximation for a compressible fluid, astrophys. J. 131, pp. 442-447 (1960).

[11] Mihaljan, J. M., A Rigorous Exposition of the Boussinesq Approximations Applicable to a Thin Layer of Fluid, Astrophys. J. 136, pp. 1126-1133, (1962).

[12] Turner, J. S., Buoyancy Effects in Fluids, p. 9 (Cambridge University Press, Cambridge, England, 1973).

[13] Rehm, R. G., Plasma motion induced by high-intensity laser heating, Phys. of Fluids 13, No. 1, pp. 921-934, (April 1970).

[14] Landau, L. D., and Lifshitz, E. M., Fluid Mechanics, Chap. 14 (Addison-Wesley Publishing Co., Reading, Mass., 1960).

[15] Williams, F. A., Combustion Theory, p. 10, (Addison-Wesley Publishing Co., Reading, Mass., 1965).

[16] Batchelor, G. K., The conditions for dynamical similarity of motions of a frictionless perfect-gas atmosphere, Quart. J. Roy. Meteor. Soc. 79, pp. 224-235, (1953).

[17] Quintiere, J., Growth of fire in building compartments, Fire Standards and Safety, Ed. A. F. Robertson, ASTM STP 614, pp. 131167 (American Society for Testing and Materials, New York, N. Y., 1977).

[18] Lamb, H., Hydrodynamics, Art. 235 (Dover Publications, New York, N. Y., 1945).

[19] Mowbray, D. E., and Rarity, B. S. H., A theoretical and experimental investigation of the phase configuration of internal waves of small amplitude in a density stratified liquid, J. Fluid Mech. 28, pp. 1-16, (1967).

[20] Yih, C.-S., Dynamics of Nonhomogeneous Fluids, Chap. 2 (The Macmillan Co., New York, N. Y., 1965).

[21] Whitham, G. B., Linear and Nonlinear Waves, pp. 421-423 (John Wiley \& Sons, New York, N. Y., 1974).

[22] Concus, P., and Golub, G. H., Use of Fast Direct Methods for the Efficient Numerical Solution of Nonseparable Elliptic Equations, S.I.A.M. J. Numer. Anal. 10, No. 6, pp. 1103-1120, (Dec. 1973).

[23] Batchelor, G. K., An Introduction to Fluid Dynamics, p. 495, (Cambridge University Press, Cambridge, England, 1967). 